\title{
XX Genotype
}

National Cancer Institute

\section{Source}

National Cancer Institute. XX Genotype. NCI Thesaurus. Code C45976.

The normal genotype of a female human. 